\title{
Adoption of strategies to improve decision support in community mental health centers
}

\author{
Shari L Hutchison ${ }^{1 *}$, Irina Karpov${ }^{1}$, Pat E Deegan², Kim L MacDonald-Wilson', James M Schuster ${ }^{1}$ \\ From 7th Annual Conference on the Science of Dissemination and Implementation in Health \\ North Bethesda, MD, USA. 8-9 December 2014
}

\section{Objective}

Assessment of specific skills acquired during training of a new practice is important in understanding adoption, but often difficult to do. This project summarizes information from a clinical skills assessment over one year post training of a program to increase treatment decision support by staff for individuals receiving mental health services.

\section{Method}

Training and implementation on a Decision Support Toolkit (Pat Deegan \& Associates) was provided and supported by a managed behavioral health care organization to interested providers. Individuals in service and staff at four community mental health centers completed a skills and practices survey where they rated how often 18 activities occurred during their clinical sessions on a Likert scale. To examine subscales, an exploratory factor analysis was conducted using the Principal Axis Factoring method for non-normally distributed data with Promax rotation to account for correlations among subscales.

\section{Results}

Surveys were completed by 41 staff at baseline, 43 at 6 months, 40 at 12 months and 300 individuals in service at baseline, 352 at 6 months, and 347 at 12 months. Three subscales were derived: Clinical (e.g., establish goals, discuss medication instructions), Empathy (e.g., offer empathy, support individual-clinician communication), and Information (e.g., provide information, educate families on mental health). Confirmatory factor analysis yielded chi-square $\mathrm{p}$-value $=0.09$ indicating a good fit. Staff ratings for Empathy were significantly higher at 12 months $\mathrm{v}$. baseline $(\mathrm{p}=0.008)$ and on average were higher than individual report $(p=0.004)$. The same pattern was seen for Information $(p=0.007)$, but on average staff ratings were lower than reported by individuals $(\mathrm{p}<0.0001)$. Clinical skills were not expected to change and did not. Discrepancies between staff and individual reports will be further investigated.

\section{Statement}

Assessment of specific skills acquired during training can be monitored and compared to report from those receiving services to confirm adoption of a new practice and discover needed improvements.

\section{Funding}

Community Care Behavioral Health Organization.

\section{Authors' details}

${ }^{1}$ UPMC Insurance Division, Community Care Behavioral Health Organization, Pittsburgh, PA 15222, USA. ²Pat Deegan PhD \& Associates, LLC, Byfield, MA 01922, USA.

Published: 20 August 2015

doi:10.1186/1748-5908-10-S1-A27

Cite this article as: Hutchison et al:: Adoption of strategies to improve decision support in community mental health centers. Implementation Science 2015 10(Suppl 1):A27.

\footnotetext{
* Correspondence: hutchisons@ccbh.com

'UPMC Insurance Division, Community Care Behavioral Health Organization, Pittsburgh, PA 15222, USA

Full list of author information is available at the end of the article
} 